OPEN ACCESS

Edited by:

David E MacHugh,

University College Dublin, Ireland

Reviewed by:

Tao Zhou,

Auburn University, United States

Xiangdong Ding,

China Agricultural University (CAU),

China

*Correspondence:

Hao Zhang

zhanghao@scau.edu.cn

Specialty section:

This article was submitted to

Livestock Genomics,

a section of the journal

Frontiers in Genetics

Received: 17 February 2019

Accepted: 24 June 2019

Published: 26 July 2019

Citation:

Hong L, Liu R, Qiao X, Wang X,

Wang S, Li J, Wu Z and Zhang H

(2019) Differential microRNA

Expression in Porcine Endometrium

Involved in Remodeling and

Angiogenesis That Contributes to

Embryonic Implantation.

Front. Genet. 10:661

doi: 10.3389/fgene.2019.00661

\section{Differential microRNA Expression in Porcine Endometrium Involved in Remodeling and Angiogenesis That Contributes to Embryonic Implantation}

\author{
Linjun Hong 1,2, Ruize Liü3 ${ }^{3}$ Xiwu Qiao ${ }^{1,2}$, Xingwang Wang ${ }^{1,2}$, Shouqi Wang ${ }^{1,2}$, Jiaqi Li $^{1,2}$, \\ Zhenfang $\mathrm{Wu}^{1,2}$ and Hao Zhang ${ }^{1,2 *}$ \\ ${ }^{1}$ National Engineering Research Center for Breeding Swine Industry, College of Animal Science, South China Agricultural \\ University, Guangzhou, China, ${ }^{2}$ Guangdong Provincial Key Laboratory of Agro-animal Genomics and Molecular Breeding, \\ College of Animal Science, South China Agricultural University, Guangzhou, China, ${ }^{3}$ Stanley Center for Psychiatric Research, \\ Broad Institute of MIT and Harvard, Cambridge, MA, United States
}

Background: In western swine breeds, up to 30\% of embryonic losses occur during early pregnancy, and the majority of embryonic losses happens during implantation. In this period, maternal recognition of pregnancy begins to occur and blastocysts undergo dramatic morphologic changes. As with other species, changes in the uterine environment plays an important role in the process of embryo implantation in pigs. Erhualian (ER) pigs, one of the Chinese Taihu swine breeds, are known to have the highest litter size in the world. Experiments demonstrated that the greater embryonic survival on gestation day (GD) 12 in Chinese Taihu pigs is one important factor that contributes to enhanced litter size. This is largely controlled by maternal genes. In this study, endometrial samples were collected from pregnant LandracexLarge Yorkshire (LL) sows (parity 3) and ER sows (parity 3) on GD12 and the expression profiles of microRNAs (miRNAs) in the endometrium were compared between ER and LL using miRNA-seq technology.

Results: A total of 288 miRNAs were identified in the pig endometrium, including 202 previously known and 86 novel miRNAs. The Kyoto Encyclopedia of Genes and Genomes pathway analysis revealed that highly abundant miRNAs might affect endometrial remodeling. Comparison between $\mathrm{LL}$ and ER sows revealed that 96 known miRNAs were differentially expressed between the two groups (including 78 up-regulated and 18 down-regulated miRNAs in ER compared to LL). Bioinformatics analysis showed that the target genes of some differentially expressed miRNAs were involved in pathways related to angiogenesis, proliferation, apoptosis, and tissue remodeling, which play critical roles in implantation by regulating endometrial structural changes and secretions of hormones, growth factors, and nutrients. Furthermore, the results demonstrated that insulin-like growth factor-1 protein expression was directly inhibited by miR-206. The lower expression of miR-206 in ER compared to LL might facilitate the angiogenesis of the endometrium during embryo implantation. 


\begin{abstract}
Conclusions: The identified miRNAs that are differentially expressed in the endometrium of ER and LL pigs will contribute to the understanding of the role of miRNAs in embryonic implantation and the molecular mechanisms of the highest embryonic survival in Chinese ER pigs.
\end{abstract}

Keywords: porcine, endometrium, microRNAs, differential expression, implantation

\section{INTRODUCTION}

Litter size has a great impact on the profitability of swine production. Prenatal mortality is the major limitation for increasing the litter size in pigs. Up to $30 \%$ of conceptuses are spontaneously lost during early pregnancy, especially on gestation days (GD) 11 to 13 (Scofield et al., 1974; Pope and First, 1985; Zavy and Geisert, 1994; Wilson et al., 1999). In contrast to rodents and primates, pigs have an extended period of preimplantation. From GD4 to GD12, the developing conceptuses undergo speedy morphologic changes (from spherical to tubular to filamentous forms) and migrate freely in the uterine cavity. On GD15, filamentous conceptuses grow to 800 to $1,000 \mathrm{~mm}$ in length and begin to attach to luminal uterine epithelium (LE) (Geisert et al., 1982; Bazer and Johnson, 2014). Thus, during protracted preimplantation, the requirement for nutrients of conceptuses is mainly dependent on uterine secretions, including glucose, amino acids, ions, enzymes, growth factors, hormones, growth factors, and other substances termed as histotroph (Spencer et al., 2006).

Chinese Taihu pigs, including Erhualian (ER), Meishan, and Fengjing breeds, are highly prolific. ER pigs are known to have the biggest litter size record in the world (Zhang, 1986). Meishan pigs were exported to western countries in the early 1980s and have been studied for more than 30 years to explore the mechanism of prolificacy. Studies found that the greater embryonic survival on GD11 to GD12 in Chinese Taihu pig is the most important factor contributing to enhanced litter size, and this is controlled primarily by maternal genes (Haley et al., 1995). At this stage, substantial changes occur in the conceptus-uterine interface, including morphologic changes in the conceptus, the onset of synthesis of estradiol by the conceptus, and the appropriate physiologic adjustments of uterus (Bazer and Johnson, 2014). Examination of individual embryos in Meishan pigs found that embryo survival was $108.1 \%$ on GD11 and $93.3 \%$ on GD12; however, in Landrace $\times$ Large Yorkshire (LL) pigs, embryo survival was $89.1 \%$ on GD11 and $49.9 \%$ on GD12 (Ashworth et al., 1997). Other studies also demonstrated that, from GD11 to GD12, the embryonic survival rate in Chinese Taihu pigs was significantly higher than in western pigs (Bazer et al., 1988; Christenson et al., 1993). Thus, it is worth further studying the molecular mechanisms underlying the differences in uterine environment changes between Chinese Taihu and western pigs.

microRNAs (miRNAs) are short (20-25 nt), endogenous, conserved, non-coding RNA molecules that play wide biological roles in transcription and translation (Carrington and Ambros, 2003; Bartel, 2004). miRNAs typically interact with target mRNAs by base pairing and destabilize or degrade their complementary
mRNA (Wu et al., 2006). They have been shown to participate in the regulation of various physiologic processes, including cellular proliferation, differentiation, apoptosis, angiogenesis, embryonic development, and reproduction control (Laurent, 2010; Nicoli et al., 2012; Rosenbluth et al., 2013). A large number of miRNAs have been shown to be associated with embryo implantation in humans and mice, such as the regulation of endometrial receptivity (Altmäe et al., 2013) and endometrial stromal cell differentiation (Qian et al., 2009), participating in human pregnancy and parturition (Montenegro et al., 2009). In pigs, Su et al. and Liu et al. reported that miRNAs play roles in porcine placental growth and functions (Su et al., 2010; Liu et al., 2015). In addition, Wessels et al. investigated the expression of miRNAs on both sides of the maternal-fetal interface in the model of implantation failure and spontaneous fetal loss in pigs and identified miRNAs that might contribute to fetal loss (Wessels et al., 2013). Thus, taken together, these results indicate the importance of miRNAs in pig reproduction. In this study, the expression profiles of miRNAs in the sow endometrium on GD12 were compared between ER and LL pigs using sequencing technology. Differentially expressed miRNAs (DEMs) were identified and miRNAs involved in reproduction were analyzed by bioinformatic analysis and experiments. Collectively, these results will help better understand the role of miRNAs in embryonic survival during implantation.

\section{MATERIALS AND METHODS}

\section{Tissue Collection}

All of the experiments involving animals were conducted according to animal ethics guidelines and approved by the Animal Care and Use Committee of South China Agricultural University (Guangzhou, China). LL and ER sows were obtained from the breeding pig farm of Guangdong Wen's Foodstuffs Group Co., Ltd. (Yunfu, China). Three LL sows (parity 3) and three ER sows (parity 3 ) were checked for estrus twice daily and artificially inseminated at the onset of estrus (day 0 ) and again $12 \mathrm{~h}$ later. After the sows were slaughtered at a local slaughterhouse on GD12, the uteri were removed rapidly and transported in an icebox to the laboratory. Pregnancy was confirmed by the presence of apparently normal filamentous conceptuses in uterine flushings. Endometrial samples were collected and stored at $-80^{\circ} \mathrm{C}$ for RNA extraction.

\section{RNA Extraction and Small RNA (sRNA) Sequencing}

Total RNA was extracted from six endometrial samples using TRIzol (Invitrogen, Carlsbad, CA, USA) according to the manufacturer's instruction. RNA purity was quantified using 
NanoDrop ND2000 spectrophotometer at 260 and $280 \mathrm{~nm}$ (Thermo Fisher Scientific, Wilmington, MA, USA), and RNA integrity was verified using an Agilent 2100 Bioanalyzer (Agilent Technologies, Palo Alto, CA, USA). The OD260/OD280 ratios of all the samples were greater than 1.8, and the RIN values were greater than 8 . Equal RNA quantities from the endometria of three pigs from the LL and ER groups were pooled. sRNA Illumina sequencing was conducted as follows: $\sim 10 \mu \mathrm{g}$ total RNA was size fractionated by Novex 15\% TBE-Urea gel and RNA fragments between 18 and 30 bases in length were isolated. The purified sRNAs were then ligated with the $5^{\prime}$-adapter. To remove unligated adapters, the ligation products (36-50 bases in length) were gel purified on Novex 15\% TBE-Urea gel. Subsequently, the RNA fragments with the adapter at the $5^{\prime}$-end were ligated with $3^{\prime}$-adapters. After gel purification on Novex 10\% TBE-Urea gel, RNA fragments with adapters at both ends (62-75 bases long) were reverse transcribed. Reverse transcription-polymerase chain reaction (RT-PCR) was used to create CDNA constructs based on the sRNA ligated with the $5^{\prime}$ - and $3^{\prime}$-adapters. This protocol gel purifies the amplified cDNA construct in preparation for loading on the Illumina Cluster Station. The cDNAs were amplified using the appropriate PCR cycles to produce sequencing libraries. Sequencing was carried out at BGI-Shenzhen, China.

\section{Sequence Analysis}

First, raw data (raw reads) were processed by custom Perl and Python scripts, raw reads contain poly-A/T/G/C, poly-N, with $5^{\prime}$-adapter contaminants, without $3^{\prime}$-adapter, or the insert tag, and low-quality reads were filtered to get clean data. Subsequently, clean reads $\geq 18 \mathrm{nt}$ were chosen as sRNA tags and mapped to reference sequence by Bowtie (Langmead et al., 2009) without mismatch to analyze their expression and distribution on the reference. Third, miRBase (release 20.0) was used as reference, and srna-tools-cli and modified software mirdeep2 (Friedländer et al., 2011) were used to obtain the potential miRNA and draw the secondary structures. The available software mirdeep2 (Friedländer et al., 2011) and miREvo (Wen et al., 2012) were integrated to predict novel miRNAs by exploring the secondary structure. At the same time, custom scripts were used to obtain the identified miRNA counts as well as base bias on the first position with certain length and on each position of all identified miRNAs, respectively.

\section{Identification of DEMs}

The procedures that determine the DEMs between LL and ER groups are shown below:

miRNA expression levels were estimated by transcript per million (TPM) with the following criteria (Wagner et al., 2012): Normalization formula: Normalized expression = Actual miRNA count/Total count of clean $\operatorname{reads}^{\star} 1,000,000$. miRNAs with a normalized expression level of less than 1 in each of the two libraries and miRNAs with an estimated probability value of less than 0.95 were removed. The fold change in the expression level and the $P$ value between two libraries were calculated from the normalized expression using the following formulas, respectively:
Fold change formula: Fold change $=\log _{2}(\mathrm{ER} / \mathrm{LL})$

$P$-value formula:

$$
p(x \mid y)=\left(\frac{N_{2}}{N_{1}}\right)^{y} \frac{(x+y) !}{x ! y !\left(1+\frac{N_{2}}{N_{1}}\right)^{(\mathrm{x}+\mathrm{y}+1)}}
$$

$N_{1}$ and $x$ represent the total count of clean reads and the normalized expression level of a given miRNA in an sRNA library of LL endometrial tissue sample, respectively. $N_{2}$ and $y$ represent the total count of clean reads and the normalized expression level of a given miRNA in an sRNA library of ER endometrial tissue sample, respectively (Audic and Claverie, 1997). Raw $P$ values were converted to adjusted $P$ values using the BenjaminiHochberg false discovery rate (Benjamini and Hochberg, 1995). The adjusted $P<0.05$ and $\mid \log _{2}$ (fold change) $\mid>1$ were set as thresholds for significantly differential expression by default.

\section{Target Gene Prediction and Functional Analysis of DEMs}

The prediction of the target genes of miRNAs was performed by RNAhybrid and TargetScan. Overlapping target genes were selected for further analysis. To reveal the target genes' potential biological functions and identify the main pathways targeted by the gene candidates, Gene Ontology (GO) and Kyoto Encyclopedia of Genes and Genomes (KEGG) pathway analyses were performed as described previously (Zhang et al., 2013). Based on the GO and KEGG database, the hypergeometric test was preformed to identify significantly enriched GO terms $(Q<0.05)$ and classify the pathway category (Boyle et al., 2004). The network of pathways based on the GO and KEGG database was constituted by ClueGO, which is a plugin in Cytoscape (http://www.cytoscape.org/).

\section{Validation of miRNA Expression via Stem- Loop Quantitative RT-PCR (qRT-PCR)}

The sRNA-seq results were validated using RNA samples from the LL $(n=3)$ and ER $(n=3)$ groups by the stem-loop qRTPCR method. A total of 16 miRNAs were selected for qRT-PCR validation. The mature miRNA and primer sequences are available in Supplementary Table S1. Briefly, for RT-PCR, the Revert Aid ${ }^{\mathrm{TM}}$ First Strand cDNA Synthesis Kit (Promega, Fitchburg, WI, USA) was adopted according to the manufacturer's instructions. Then, RT-PCR was performed with SYBR ${ }^{\circledR}$ Premix Ex Taq ${ }^{\mathrm{TM}}$ (Toyobo) on ABI PRISM ${ }^{\circledR} 7500$ Sequence Detection System. Porcine U6 snRNA was used as an internal control and all reactions were run in triplicate. PCR profiles were one cycle at $95^{\circ} \mathrm{C}$ for $5 \mathrm{~min}$ followed by $40 \times\left(95^{\circ} \mathrm{C}\right.$ for $15 \mathrm{~s}, 65^{\circ} \mathrm{C}$ for $15 \mathrm{~s}$, and $72^{\circ} \mathrm{C}$ for $\left.32 \mathrm{~s}\right)$. The relative expression levels were calculated using the $2^{-\Delta \Delta \mathrm{Ct}}$ method. Fold change ( $\log _{2}$ ratio) was used to show the differential expression of miRNA in LL and ER.

\section{Dual-Luciferase Reporter Assays}

For luciferase reporter experiments, the pmirGLO dualluciferase reporter vector (Promega) housing the $3^{\prime}$-untranslated 
region (UTR) of insulin-like growth factor-1 (IGF-1), which was XhoI and XbaI cloned to the $3^{\prime}$-end of the Renilla gene, was used to examine the effect of miR-206 on Renilla production. IGF-1 3'-UTR vector (pmirGLO-IGF1) containing the miR-206 binding site (CATTCC) was constructed by RT-PCR using specific primers (forward primer 5'-CCGCTCGAGCAGGAAACAAGA ACTACAG-3' and reverse primer 5'-GCTCTAGACAACAGCA ATCTACCAACT-3'). Meanwhile, IGF-1 3'-UTR-Mutant vector (pmirGLO-IGF1-Mutant) with a mutated miR-206 binding site (GTAAGG) was also constructed. The miR-206 mimics and its mutant mimics (miR-206_mut) were designed and synthesized by GenePharma Biotech Co. (Shanghai, China). In the dualluciferase assays, PK15 cells were cultured in DMEM complete medium (Hyclone, Logan, UT, USA) and then plated onto a 96-well plate. The miR-206 mimics, mutant miR-206mimics, or negative control (NC) were co-transfected into cells with 3'-UTR dual-luciferase vector using Lipofectamine 2000 (Invitrogen, Shanghai, China). Cells were collected $24 \mathrm{~h}$ after transfection, and assayed with the Dual-Luciferase Reporter Assay System (Promega). Three replicates were performed for each transfection.

\section{Lentivirus Preparation and Administration}

The pri-miR-206 expression lentivirus vector (H1-MCS-CMVEGFP) and the NC lentivirus vector were purchased from GenePharma Biotech. Virus titration and infection efficiency were measured by the fluorescence method as lentiviral vectors expressed enhanced green fluorescent protein in infected cells. According to the results of a preliminary experiment, the titer of lenti-pri-miR-206 used for experiments was $1 \times 10^{7} \mathrm{TU} / \mathrm{ml}$. The lentivirus vectors were transfected into porcine skeletal muscle satellite cells (SCs) with a titer of $1 \times 10^{7} \mathrm{TU} / \mathrm{ml}$ in the presence of polybrene $(5 \mu \mathrm{g} / \mathrm{ml})$. Cells were collected $72 \mathrm{~h}$ after transfection, and total RNA and protein were extracted for further experiments.

\section{Western Blot Analysis}

Protein lysates were generated using the mammalian protein extraction reagent RIPA (Beyotime, Shanghai, China). The concentration of extracted total protein from each sample was calculated using the BCA Protein Assay Kit (Thermo Pierce, Rockford, IL, USA). The equivalent protein for each sample was loaded into a $10 \%$ sodium docedyl sulfate-polyacrylamide gel electrophoresis and fractionated, and the denatured proteins were subsequently transferred from gel to a polyvinylidene fluoride membrane (Millipore, Billerica, MA, USA) by a MiniPROTEAN Tube Cell instrument (Bio-Rad, Hercules, CA, USA). The membranes were incubated with antibodies (IGF-1, ab9572, Abcam; glyceraldehyde 3-phosphate dehydrogenase, ab8245, Abcam) overnight at $4^{\circ} \mathrm{C}$ and then with horseradish peroxidaseconjugated goat anti-rabbit secondary antibody for $1 \mathrm{~h}$ at room temperature. The enhanced chemiluminescence substrate (Beyotime) was used to visualize the band, and a picture was captured by an imaging system (UVP, Upland, CA, USA). Finally, the quantification analysis was performed by ImageJ 1.45 software (NIH Image).

\section{Statistical Analysis}

Data from the results of qRT-PCR, dual-luciferase reporter assays, and Western blot analysis were analyzed using SPSS version 18.0 (SPSS, Inc., Chicago, IL, USA). Paired $t$ tests and two-way analyses of variance were performed to analyze the relative expression of miRNAs, the luciferase activity, and the intensity of the protein band in Western blot analysis. $P<0.05$ was considered statistically significant.

\section{RESULTS}

\section{Overview of the Squences Generated by Illumina Sequencing}

sRNA libraries were generated from a total of six samples from ER and LL sows on GD12. After removing low-quality reads and adaptor sequences, a total of 9,104,438 and 12,881,211 clean reads were obtained from ER and LL samples, respectively. The sRNA annotation is presented in Supplementary Table S2. The results of sRNA annotation showed that known miRNAs accounted for $48.64 \%$ and $56.96 \%$ of the total clean reads in ER and LL, respectively (Supplementary Table S2). The distribution of sequence lengths was similar between ER and LL libraries (Figure 1A). The number of 20 to 23 nt sequences was significantly greater than that of shorter or longer sequences, and almost half of the sequences in LL (47.03\%) and $39.33 \%$ sequences in ER are $22 \mathrm{nt}$.

In total sRNA reads, $20,689,836$ common sequences were obtained in LL and ER, accounting for $94.11 \%$ of the total sequence reads in the two libraries (Figure 1B). In unique sRNA reads, 63,548 common sequences were obtained in LL and ER, accounting for $7.69 \%$ of the total reads in the two libraries (Figure 1C), and 361,418 (43.71\%) and 401,914 (48.61\%) specific sequences were obtained from LL and ER, respectively (Figure 1C).

\section{Sequence Variants and Editing of Bases in the Seed Region of miRNAs}

Sequencing data analysis revealed that the majority of identified miRNAs showed length and sequence heterogeneity in the porcine endometrial tissue. The length variations occurred largely in the $3^{\prime}$-end of miRNAs, mainly in the form of terminal reductions or additions of nucleotides. In ER, miR-128, miR-187, miR-18b, miR-190, miR-196a, miR-206, miR-215, miR-2476, miR-326, miR-338, miR-676, and miR-758 had variants only at the $3^{\prime}$-end, whereas, in LL, miR-105, miR-129b, miR-149, miR-153, miR-190, miR-208b, miR-216, miR-450a, miR-450c-5p, miR-499-5p, miR503, and miR-95 had variants only at the $3^{\prime}$-end. In addition, 11 and 10 miRNAs in the ER and LL libraries, respectively, were mutated by only one nucleotide in the $5^{\prime}$-end, but they had several $3^{\prime}$-end variants (Supplementary Tables S3 and S4). Similarly, previous studies also revealed the length variations of miRNAs in other porcine tissues ( $\mathrm{Li}$ et al., 2010; Nielsen et al., 2010; Li et al., 2011). Such variants might be from altered miRNA processing, prioritized degradation at miRNA ends, or post-transcriptional modifications, including RNA editing (Aravin and Tuschl, 2005). These end-sequence variations are interesting as they may allow 

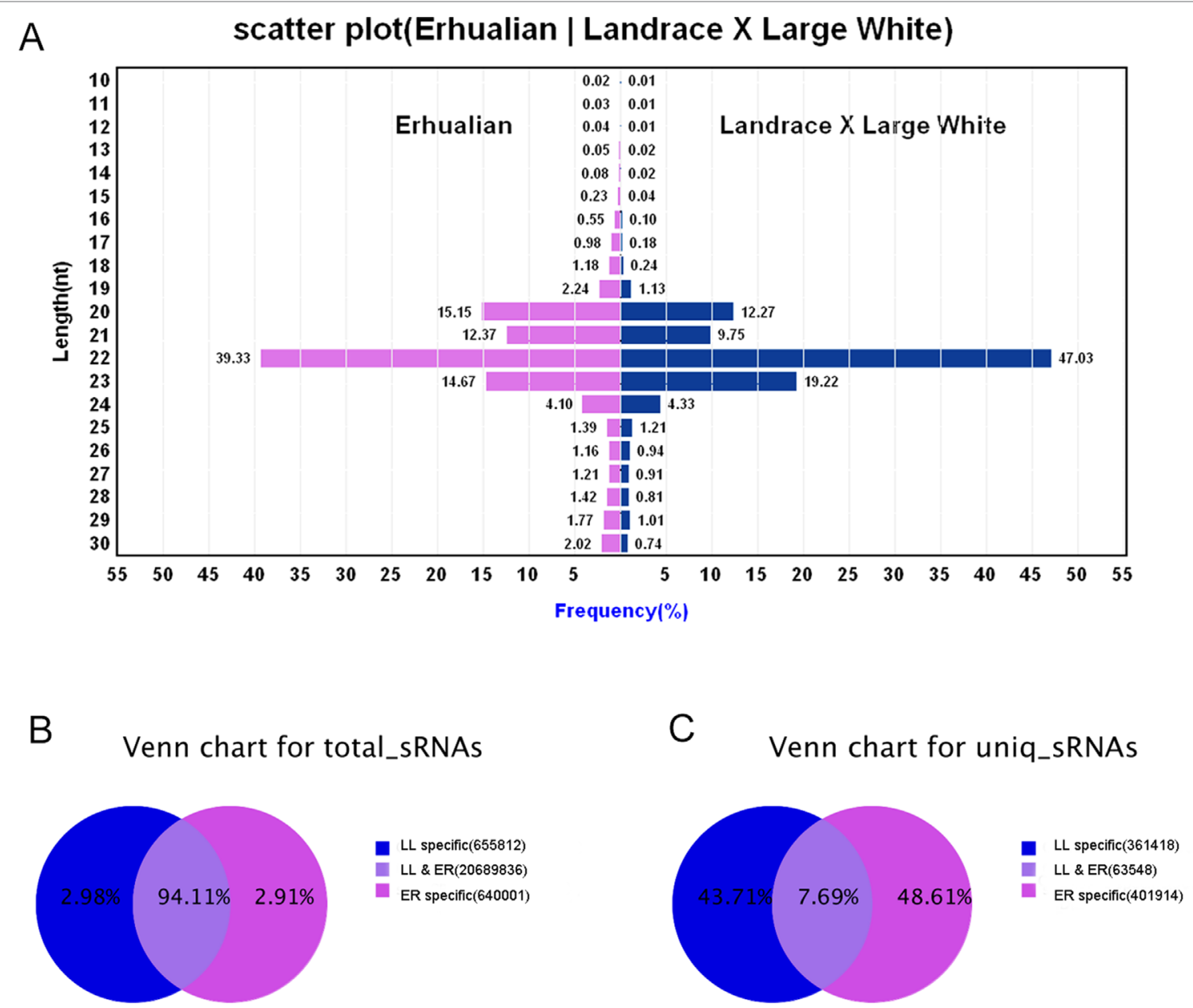

FIGURE 1 | Overview of the sequences generated by Illumina sequencing. (A) Sequence length distributions of the two libraries. The length distributions peaked at $22 \mathrm{nt}$, which is expected for miRNAs' length. (B) Number of total sRNA tags between the two libraries. (C) Number of unique sRNA tags between the two libraries.

miRNA variants to play different roles by influencing the miRNAtarget mRNA hybrid duplex structure (Jazdzewski et al., 2009). The nucleotides at positions 2 to 8 of a mature miRNA are known as the seed region. The seed region binds to a target site in the $3^{\prime}$-UTR of the target mRNA by complementarities and is highly conserved. The target of an miRNA may alter due to change in the nucleotides in the seed region. Editing of bases in the seed region of miRNAs has been reported to occur frequently (Kawahara et al., 2007; Liu et al., 2008). In the present analysis, miRNAs that might have seed editing can be distinguished by matching unannotated sRNA with porcine mature miRNAs from miRBase 20.0. Fortynine and 62 mature miRNAs in ER and LL had a single nucleotide substitution in the seed region, respectively (Supplementary Table S5). The observed occurrence for each possible substitution is summarized in Supplementary Table S5 for ER and LL samples, respectively. In ER, the most frequent substitutions were T-to- $\mathrm{C}$ (22.2\%), A-to-G (20.7\%), and G-to-A (13.6\%), whereas, in LL, the most frequent substitutions were T-to-G (18.1\%), T-to-A (15.0\%), and G-to-T (14.5\%; Figure 2). Although the most frequent substitutions were different between ER and LL libraries, C-to-A (0.4\% in ER; $2 . \%)$ and C-to-G (0.4\% in ER; $2 . \%)$ were the substitutions with the lowest frequency in both ER and LL libraries. In porcine adipose tissue samples, similar results were also reported (Li et al., 2011). Interestingly, abundant miRNAs (ssc-let-7a, ssc-mir-143, ssc-let-7f, ssc-mir-21, and ssc-mir-378) also had higher editing probability (Supplementary Table S5). This indicates that highly expressed miRNAs targeted more genes.

\section{Expression Profiling of miRNAs}

In the second part of the present analysis, the global expression profile of endometrial miRNAs on GD12 in LL and ER pigs was determined. A total of 288 miRNAs were identified in the pig endometrium, including 202 known miRNAs and 86 novel miRNAs (Supplementary Table S6). Among the known miRNAs, 200 miRNAs were co-expressed in LL and ER and 2 miRNAs (ssc-miR-124a and ssc-miR-450c-3p) were specifically expressed in ER. Among the novel miRNAs, 38 miRNAs were co-expressed in LL and ER and 19 and 28 miRNAs were specifically expressed in LL and ER, respectively.

The 20 most highly expressed miRNAs in LL and ER libraries are listed in Table 1. Among them, 14 highly expressed miRNAs 


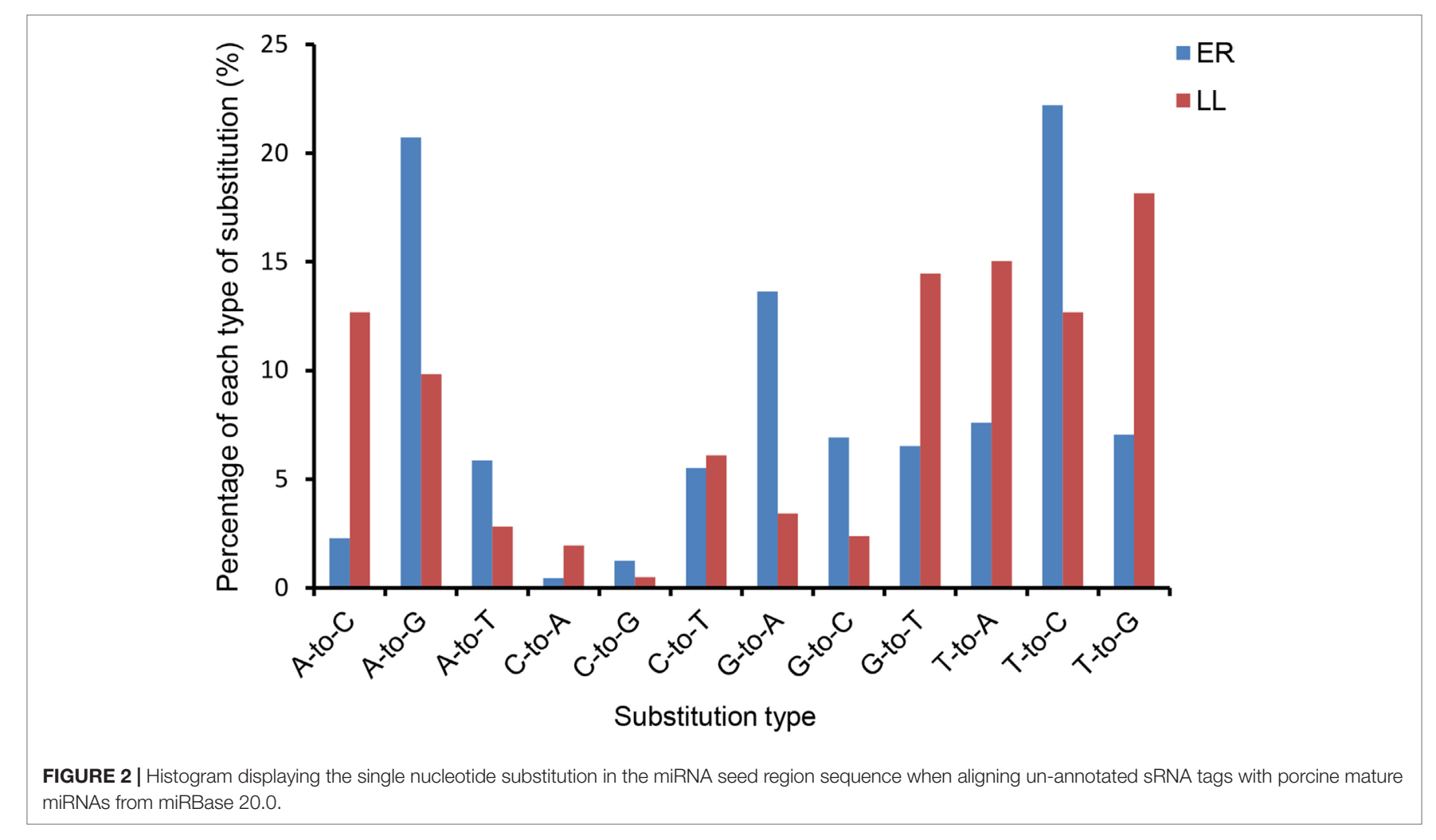

TABLE 1 | Top 20 miRNAs in LL and ER.

\begin{tabular}{|c|c|c|c|c|c|}
\hline miRNA-name & Average TPM in LL & Rank in LL & miRNA-name & Average TPM in ER & Rank in ER \\
\hline ssc-let-7a & 1289290 & 2 & ssc-let-7a & 836090 & 2 \\
\hline ssc-let-7f & 467190 & 4 & ssc-let-7f & 317357 & 4 \\
\hline ssc-miR-30a-5p & 313695 & 5 & ssc-miR-30a-5p & 92442 & 9 \\
\hline ssc-miR-148a & 290344 & 6 & ssc-miR-148a & 31042 & 18 \\
\hline ssc-miR-10a & 242124 & 7 & ssc-miR-10a & 112910 & 8 \\
\hline ssc-miR-378 & 184397 & 10 & ssc-miR-378 & 213166 & 5 \\
\hline ssc-miR-30d & 138588 & 11 & ssc-miR-30d & 63006 & 10 \\
\hline ssc-miR-34c & 102496 & 12 & ssc-miR-34c & 13563 & 31 \\
\hline ssc-miR-140* & 88368 & 13 & ssc-miR-140* & 21112 & 22 \\
\hline ssc-miR-103 & 79372 & 14 & ssc-miR-103 & 32711 & 17 \\
\hline ssc-let-7g & 78468 & 15 & ssc-let-7g & 38160 & 16 \\
\hline ssc-miR-206 & 41179 & 20 & ssc-miR-206 & 233 & 128 \\
\hline ssc-miR-26a & 40215 & 22 & ssc-miR-26a & 47934 & 11 \\
\hline ssc-miR-101 & 34754 & 23 & ssc-miR-101 & 41815 & 12 \\
\hline ssc-miR-196b-5p & 30481 & 27 & ssc-miR-196b-5p & 27714 & 19 \\
\hline ssc-miR-199a* & 21283 & 32 & ssc-miR-199a* & 39465 & 13 \\
\hline ssc-miR-199a-3p & 21236 & 33 & ssc-miR-199a-3p & 39427 & 14 \\
\hline ssc-miR-199b* & 21236 & 34 & ssc-miR-199b* & 39426 & 15 \\
\hline
\end{tabular}

The list shows the top 20 abundant miRNAs in $L L$ and ER, respectively.

The star is part of the MiRNA name. 
were the same in LL and ER. Thus, the predicted target genes of 14 common miRNAs were chosen for functional analysis. GO analysis (ClueGo network of GO terms) showed that they were mainly involved in the "cellular protein metabolic process," "regulation of macromolecule biosynthetic process," and "anatomical structure morphogenesis" (Supplementary Figure S1). The KEGG pathway analysis (ClueGo network of pathways) indicated that the predicted target genes were mainly enriched in "Apoptosis," "Autophagy," "Ubiquitin-mediated proteolysis," "Longevityregulating pathway," "AMPK signaling pathway," "Regulation of actin cytoskeleton," "Focal adhesion," "ECM-receptor interaction," "Rap1 signaling pathway," "FoxO signaling pathway," "mTOR signaling pathway," and "MAPK signaling pathway" (Figure 3).

\section{Comparative Analysis of DEMs Between ER and LL}

These DEMs between LL and ER libraries are listed in Supplementary Table S7, and in total, 96 known and 68 novel significantly DEMs were identified between LL and ER groups. Of the 96 differentially expressed known miRNAs, 78 were up-regulated and 18 were downregulated in ER compared to LL (Figure 4A). miR-206 ranked the top [fold change $\log _{2}(E R / L L)=-6.96$ ] among DEMs that were expressed in both LL and ER. Of the differentially expressed novel miRNAs, 43 were up-regulated and 25 were down-regulated in ER than in LL (Figure 4B).

\section{Validation of Sequencing Results by qRT-PCR}

The stem-loop qRT-PCR assay was used to specifically detect mature miRNAs. U6 snRNA was selected as the reference gene. Sixteen miRNAs were chosen for validation by qRT-PCR and the primers used are listed in Supplementary Table S1. The expression patterns for the 16 miRNAs were consistent with those in sequencing data (Figure 5).

\section{Functional Annotation of DEMs in Endometrial Tissue Samples}

To evaluate the biological functions of these DEMs, target genes of DEMs were predicted by RNAhybrid and TargetScan, and the KEGG pathway analysis of these target genes was performed. Thus, 275 significantly enriched signaling pathways were obtained, such as with "VEGF signaling pathway" (Angiogenesis-related pathway), "Toll-like receptor signaling pathway" (Immune-related pathway), "Regulation of actin cytoskeleton" (Tissue remodelingrelated pathway), and "MAPK signaling pathway, TGF- $\beta$ signaling pathway and Apoptosis" (Proliferation and apoptosis) in the top 25 signaling pathways (Figure 6). In addition, some other target genes were annotated to reproduction-associated pathways, including "Steroid hormone biosynthesis," "Progesterone-mediated oocyte maturation," "Steroid biosynthesis," "GnRH signaling pathway," and "p53 signaling pathway."

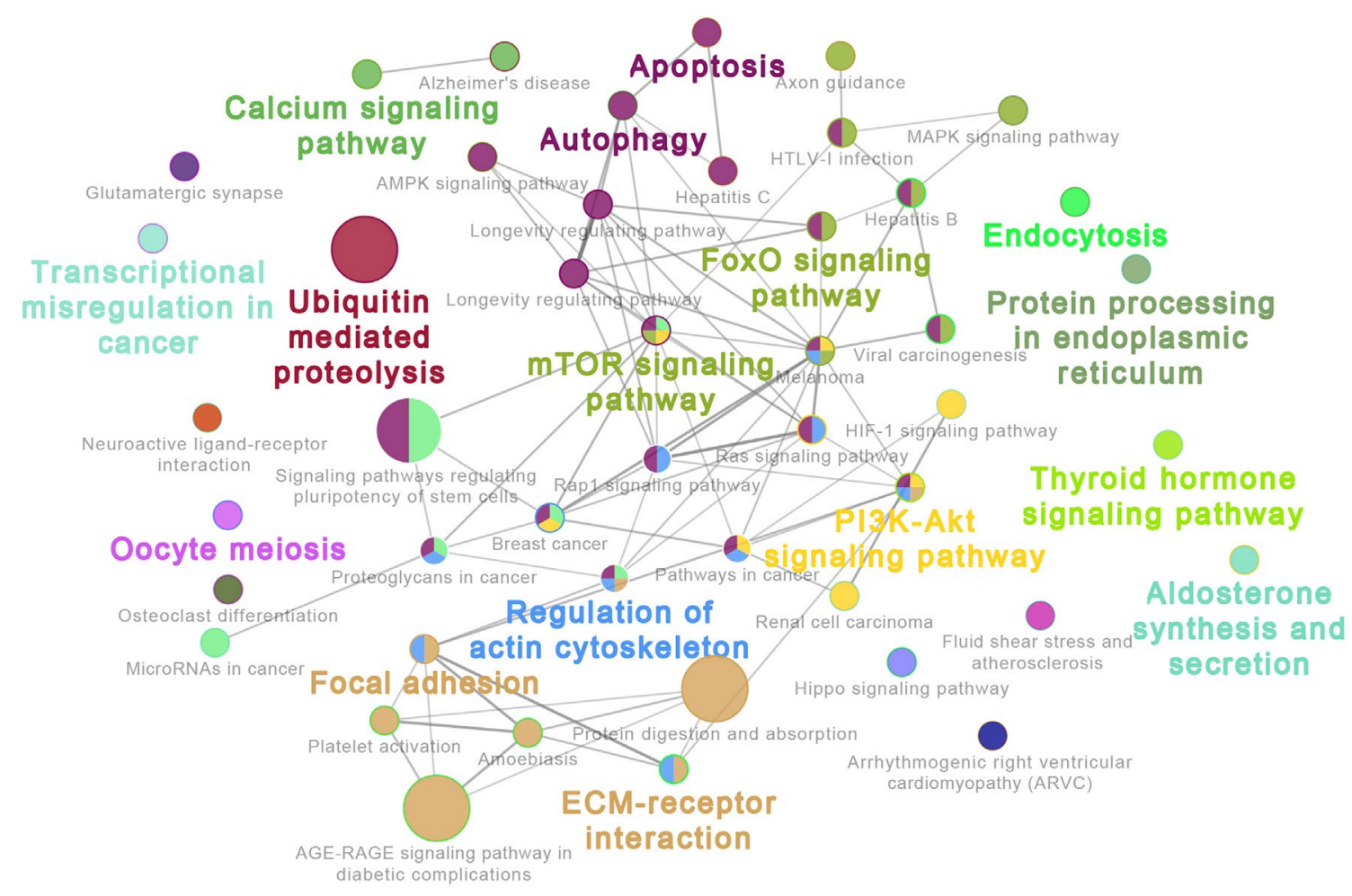

FIGURE 3 | ClueGo network of pathways. Each node represents a pathway. The enrichment significance of pathway is reflected by the size of the nodes. Node color represents the class that they belong to. Mixed coloring means that the specific node belongs to multiple classes. 

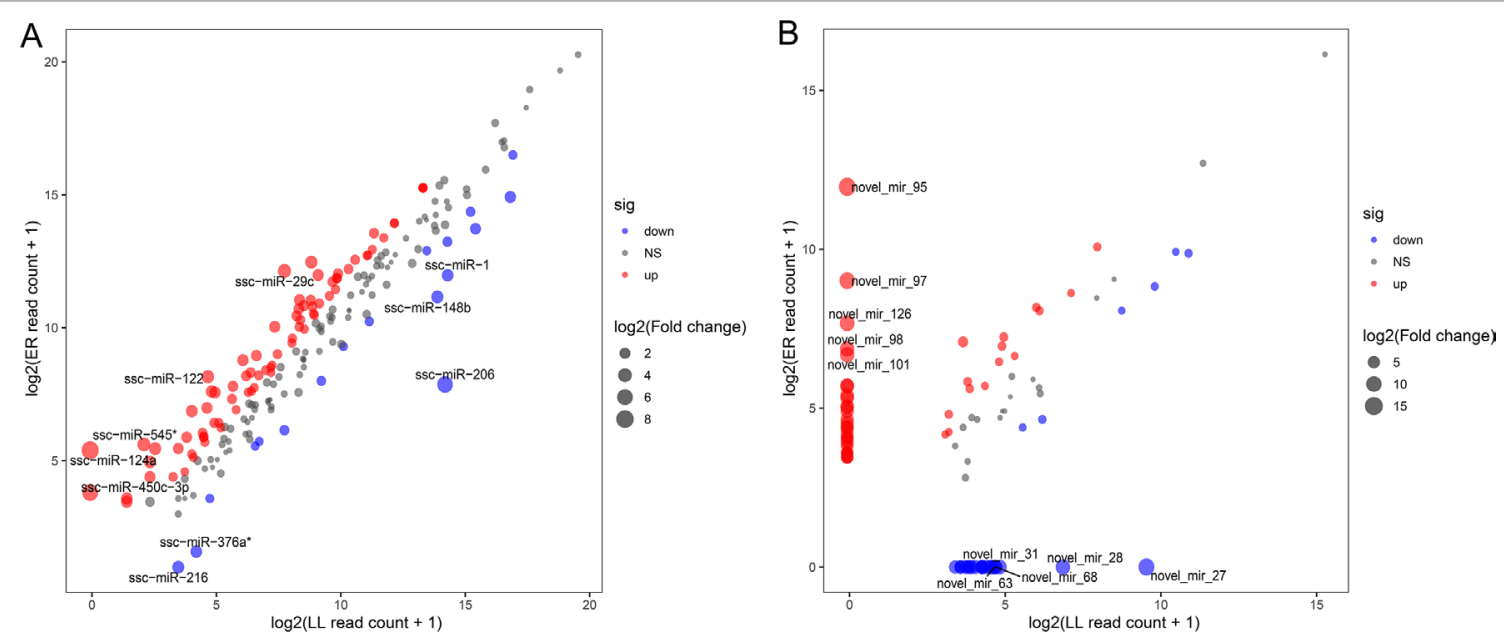

FIGURE 4 | Differential expression of porcine known (A) and novel (B) miRNAs between ER and LL. Each point in the figure represents the log ${ }_{2}($ ER/LL read count +1 ) of an miRNA. Red points represent miRNAs with $\log _{2}(\mathrm{ER} / \mathrm{LL})>1$ and adjusted $P<0.05$, blue points represent miRNAs with $\log _{2}(\mathrm{ER} / \mathrm{LL})<-1$ and adjusted $P<0.05$, and green points represent miRNAs with $1>\log _{2}(E R / L L)>-1$. The size of points shows the value of $\log _{2}(E R / L L)$.

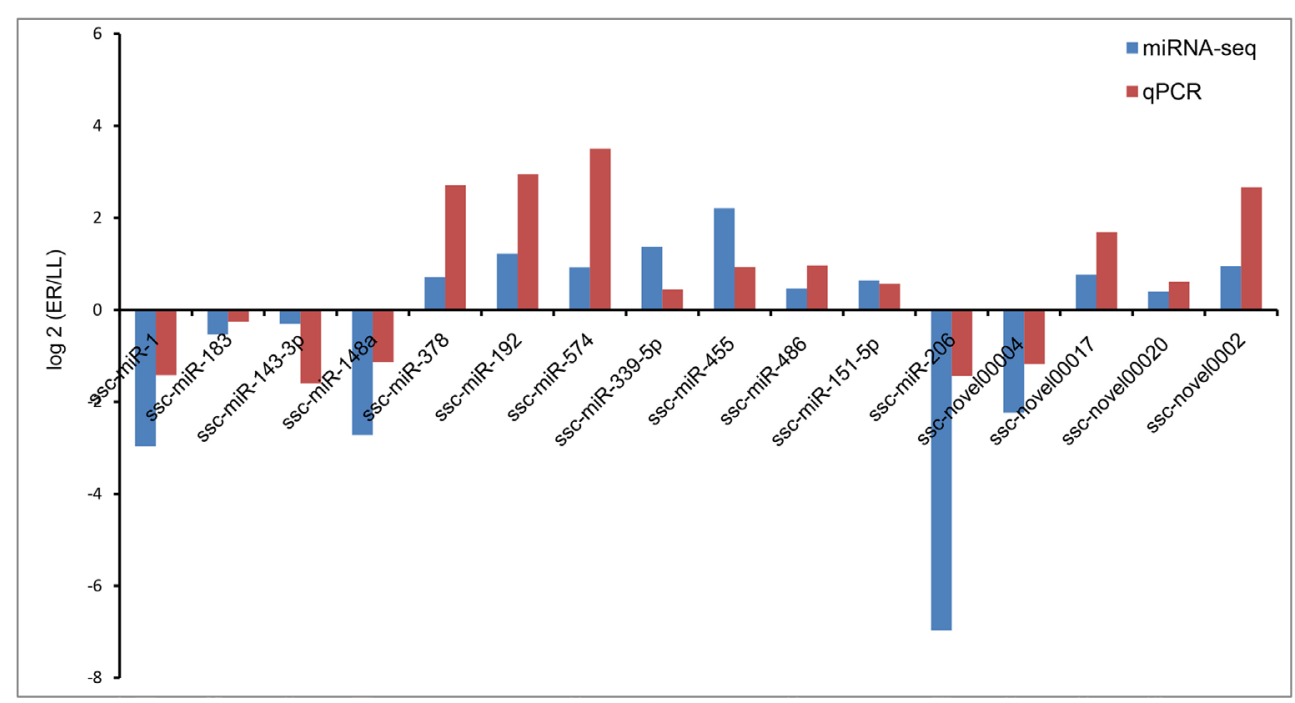

FIGURE 5 | Validation of deep sequencing results via real-time qRT-PCR.

\section{miR-206 Directly Targeted 3'-UTR of IGF-1 and Inhibited its Protein Expression}

miR-206 was the top DEM, and it was expressed in both LL and ER pigs. To uncover the function of miR-206, the potential targets of miR-206 in the data bank (www.targetscan. org; Figure 7A) was examined, and IGF-1 was identified as a prime target, with a highly conserved complementary miR-206-binding site in its $3^{\prime}$-UTR across mammals from humans to pandas (Figure 7B). As shown in Figure 7, the dual-luciferase reporter assay system showed a significant reduction of the Renilla/firefly luciferase ratio in the wildtype miRNA mimic compared to the mutant-type group.
However, miR-206 had no appreciable inhibitory effect on a mutated IGF-1 3'-UTR dual-luciferase construct (Figures 7C, D). These results demonstrate the specific inhibition of IGF-1 expression by miR-206. miRNA regulates gene expression at the transcriptional level or at translational levels. To determine the regulation mechanism of miR-206, qRT-PCR and Western blot analysis were performed. Although no significant inhibition was detected at the IGF-1 mRNA level in porcine skeletal muscle SCs that were infected with pri-miR-206 expression lentivirus (Figures $\mathbf{7 E}, \mathbf{F}$ ), the inhibitory effect of miR-206 on IGF-1 protein expression was determined by Western blot analysis (Figure 7G). Therefore, it was confirmed 


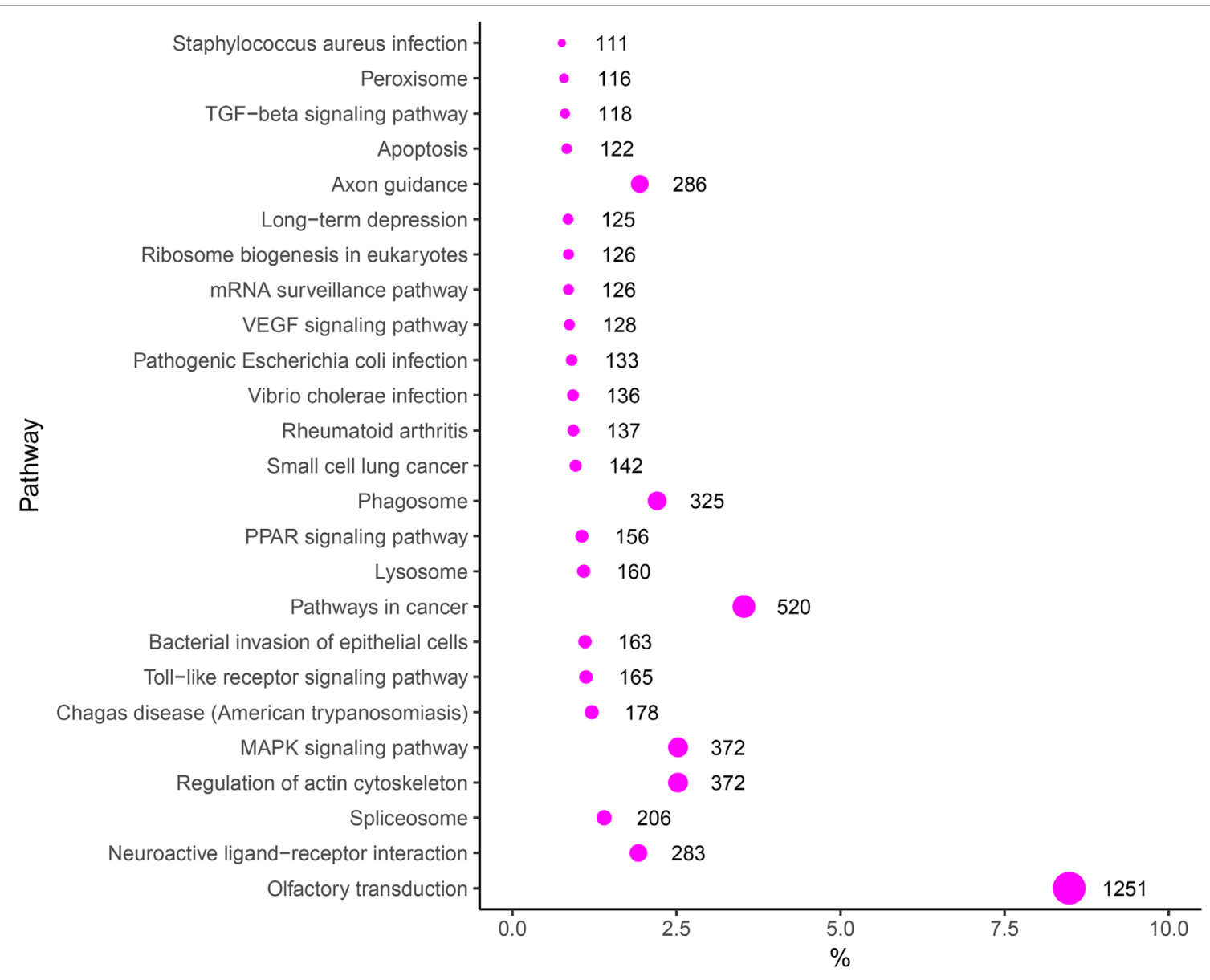

FIGURE 6 | KEGG enrichment scatter plot of the targets of DEMs.

that miR-206 directly targeted 3'-UTR of IGF-1 and inhibited its protein expression but not its mRNA transcription.

\section{DISCUSSION}

The miRNA system is a huge regulatory network of cellular processes, with a single miRNA being able to posttranscriptionally silence multiple mRNAs, while each mRNA can be targeted by numerous miRNAs (Friedman et al., 2008; Lu and Clark, 2012). In humans, more than $30 \%$ of the mRNAs are predicted to be miRNA targets (Griffiths-Jones et al., 2007). Recently, some miRNAs were found to be associated with endometrial receptivity, embryo development, and implantation (Ariel et al., 2011; Liu et al., 2016). In the present study, the sRNA profiles of endometrial tissues from ER and LL swine endometrium on GD12 using sequencing technology were compared to understand the miRNA-mediated regulation of embryo implantation. Our studies revealed the differential expression of 96 known miRNAs and 68 novel miRNAs in ER and LL endometrium, and the identification of miRNAs and target genes may be useful to develop new techniques and strategies for improving embryonic survival during implantation.

\section{Highly Abundant miRNAs Might Affect Endometrial Remodeling}

ssc-miR-143-3p, ssc-let-7a, and ssc-miR-21 were the top three miRNAs that were highly expressed in both LL and ER libraries. They were also found to be highly expressed in the endometrium of Meishan and Yorkshire pigs during early gestation in a recent published paper ( $\mathrm{Li}$ et al., 2018). Mu et al. found that miR-143-3p inhibits proliferation and induced apoptosis in human hypertrophic scar fibroblast cells, and it also inhibited extracellular matrix production-associated protein expression (Mu et al., 2016). Several other studies also demonstrated that miR-143-3p suppressed proliferation and induced apoptosis in different carcinoma cells (He et al., 2016; Chen et al., 2017); thus, the highly expressed miR-143-3p in the porcine endometrium might also play a role in regulating the proliferation and apoptosis of endometrial cells. For let-7a, a functional investigation also revealed that it suppressed the proliferation of endometrial carcinoma (Liu et al., 2013b), and another study demonstrated that it markedly suppressed the proliferation, migration, and invasion of gastric cancer cells by down-regulating PKM2 (Tang et al., 2016). Furthermore, let-7a is involved in regulating the implantation process by the modulation of the expression of integrin- $\beta 3$ and mucin 1 (Liu et al., 2012; Inyawilert et al., 2015). 
A

Position 154-160 5' AAAUACACAAGUAAACAUUCCA... of IGF1 3'UTR

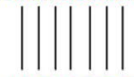

ssc-miR-206

3' GugugugAagGAAUGUAAGGU

C

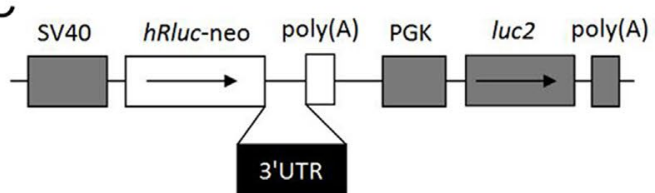

B

Pig 5'

AAAUACACAAGUAAACAUUCCAACAU

Human

Mouse

Rat

Dog

Rabbit

Horse

Chimpanzee

Baboon

panda

AAAUACACAAGUAAACAUUCCAACAU

AAAUAUACAAGUAAACAUUCCAACAU

AAAUACGCAAGUAAACAUUCCAACGU

AAAUACACAAGUAAACAUUCCAAUAU

AAAUACACAAGUAAACAUUCCAACAU

AAAUACAUAAGUAAACAUUCCAACAU

AAAUACACAAGUAAACAUUCCAACAU
AAAUACACAAGUAAACAUUCCGACAU

AAAUACGCAAGUAAACAUUCCAACAU

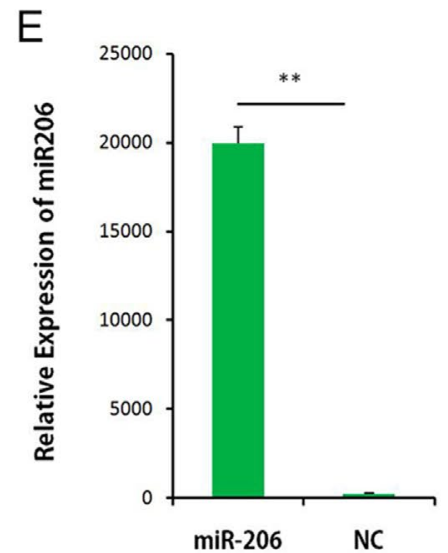

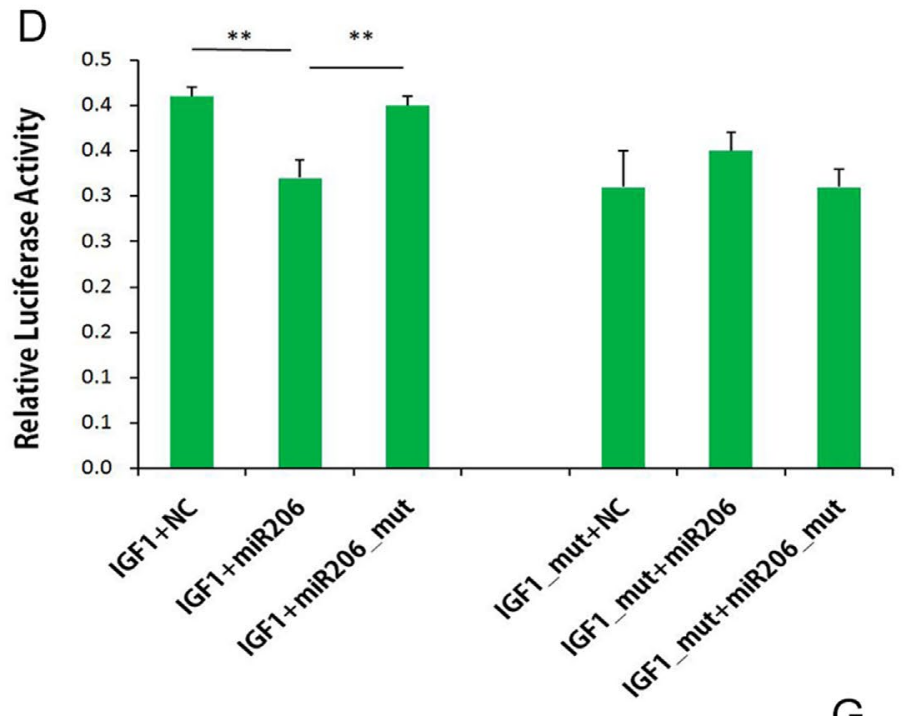

$\mathrm{F}$

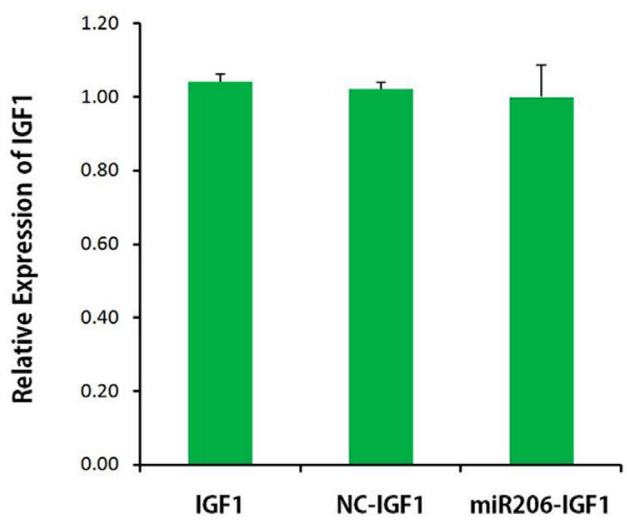

G IGF1
Cell NC

miR-206

GAPDH

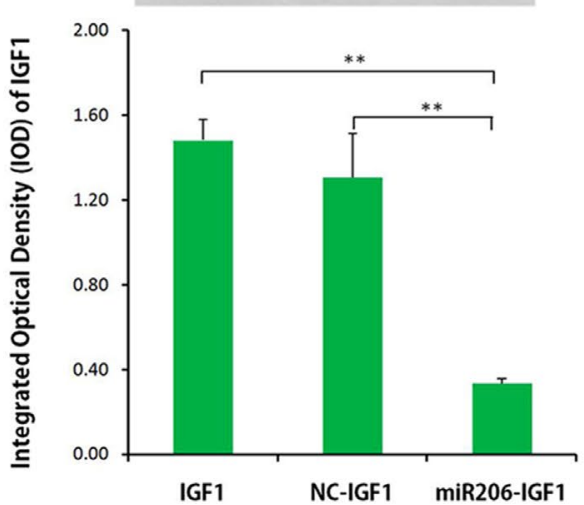

FIGURE 7 | miR-206 targets the 3'-UTR of IGF-1. (A) Predicted binding site of miR-206 in the 3'-UTR of IGF-1. (B) Binding site of miR-206 is highly conserved among mammals. (C) IGF-1 3'-UTR was inserted into the pmirGLO dual-luciferase reporter vector at the $3^{\prime}$-end of the Renilla luciferase gene (hRluc). (D) IGF-1 3'-UTR or IGF-1 3'-UTR-Mutant construct was co-transfected with miR-206, miR-206_mut, or NC, as indicated, into PK cells, and normalized Renilla luciferase activity was determined. (E) Expression of miR-206 in the porcine skeletal muscle SCs infected with pri-miR-206 expression lentivirus or NC lentivirus. (F) Expression of IGF-1 mRNA in SCs and SCs infected with pri-miR-206 expression lentivirus or NC lentivirus. (G) Expression of IGF-1 protein in SCs and SCs infected with primiR-206 expression lentivirus or NC lentivirus. Results are mean \pm SD (three independent replicates per group). ${ }^{*} P<0.05 ;{ }^{* *} P<0.01$ (Student's $t$ test). 
In addition, miR-21 has been causally linked to cellular proliferation, apoptosis, and migration in a wide variety of cancers (Asangani et al., 2008; Frankel et al., 2008). Previous studies have suggested that miR-21 was involved in embryo implantation in mouse (Hu et al., 2008), and a recent study provided evidence that miR-21 expressed in extracellular vesicles is very important in preimplantation embryo development (Lv et al., 2018). The KEGG pathway analysis of common miRNAs indicated that the predicted target genes were enriched in 1) cell self-renewal and degradation, including "Apoptosis," "Autophagy," "Ubiquitinmediated proteolysis," "Longevity-regulating pathway," and "AMPK signaling pathway"; 2) cell motility, including "Regulation of actin cytoskeleton," "Focal adhesion," "ECM-receptor interaction," and "Rap1 signaling pathway"; and 3) cell proliferation and differentiation, including "FoxO signaling pathway," "mTOR signaling pathway," and "MAPK signaling pathway," Based on these results, it can be inferred that most highly abundant miRNAs in porcine endometrium mainly played important roles in regulating endometrial remodeling at the time of implantation.

\section{Differentially Expressed Known miRNAs Related to Proliferation and Angiogenesis}

Of the 96 differentially expressed known miRNAs, more than $80 \%$ were up-regulated in ER compared to LL sows; among them, sscmiR-29c was the top miRNA that was expressed in both breeds (Supplementary Table S7). A recent study demonstrated that miR-29c affects human endometrial cells by suppressing cell proliferation and invasion as well as promotes cell apoptosis by inhibiting c-Jun expression (Long et al., 2015). It was also shown previously that miR-29c inhibited cell proliferation and induced apoptosis in many types of carcinoma cells (Wang et al., 2011; Liu et al., 2013a). Similarly, ssc-miR-214, which was among the top five miRNAs up-regulated in ER compared to LL sows (Supplementary Table S7), also played a role in promoting apoptosis and suppressing cell proliferation in several types of cells (Feng et al., 2011; Yang et al., 2013; Zhang et al., 2014). These results indicate that the markedly higher expression of miRNAs in ER than in LL (i.e., miR-29c and miR-214) could also play a key role in inhibiting endometrial cell proliferation and invasion, which could contribute to developing a more stable uterine environment. The results are also consistent with the authors' previous findings, which also revealed that the endometrium of ER pigs had a lower growth-promoting ability (Zhang et al., 2013). Strong evidence has shown that increased prolificacy of Chinese Taihu pigs might be due to an increased embryonic survival resulting from the more stable uterine environment and increased uterine receptivity (Stroband et al., 1992; Youngs et al., 1993; Youngs et al., 1994).

Pathway analyses can provide a better understanding of the molecular functions and biological processes of target genes. Among the target genes of differentially expressed known miRNAs, some KEGG pathways that are important for reproduction were significantly enriched. Notably, the mitogen-activated protein kinase (MAPK) signaling pathway, the Toll-like receptor signaling pathway, the peroxisome proliferator-activated receptor (PPAR) signaling pathway, the vascular endothelial growth factor (VEGF) signaling pathway, and the transforming growth factor- $\beta$ (TGF$\beta$ ) signaling pathway were in the top 25 signaling pathways. The MAPK signaling pathway is involved in the regulation of human endometrial cell proliferation (Park et al., 2017; Zhang et al., 2018). In another study, research data indicated that the activation of the MAPK signaling pathway can increase the proliferation of porcine uterine LE cells and may affect implantation in early pregnancy in pigs (Lim et al., 2018). In addition, the PPAR and TGF- $\beta$ signaling pathways were also related to cell proliferation and had influence on implantation (Lim and Dey, 2000; Li et al., 2004; Chang et al., 2008; Tsang et al., 2013; Cheng et al., 2017; Song et al., 2018). The Toll-like receptor signaling pathway is responsible for innate immune responses, and studies provide evidence that this pathway takes part in implantation by regulating trophoblast cells' adhesion to endometrial cells (Montazeri et al., 2016). Studies provide evidence that the VEGF signaling pathway is known as the regulator of several endothelial cell functions, including mitogenesis, permeability, vascular tone, and the production of vasoactive molecules (Giles, 2001). Previous studies also indicated that it plays important roles in implantation and maintenance of pregnancy (Das et al., 1997; Halder et al., 2000; Möller et al., 2001; Hannan et al., 2011). Collectively, these pathway analyses illustrate some of the possible roles of highly expressed miRNAs in reproduction.

IGF-1 can regulate endothelial cell migration and promote angiogenesis (Shigematsu et al., 1999). In the human endometrium, it was found that IGF-1 participates in the maintenance of an angiogenic phenotype by inducing VEGF expression (Bermont et al., 2000). Furthermore, a recent study reported that IGF-1 is a critical determinant of neonatal porcine uterine development (George et al., 2018). Our results demonstrated that IGF-1 protein expression was directly inhibited by miR-206, which were highly expressed in LL and lowly expressed in ER. This suggests that the low expression of miR-206 in ER might facilitate the angiogenesis of endometrium during peri-implantation, but further studies are required to verify this hypothesis.

\section{CONCLUSIONS}

In summary, Illumina sequencing was used to identify 288 distinct miRNAs, consisting of 202 previously reported and 86 novel miRNAs, from porcine endometrium in two different reproduction capacity breeds. In a comparison of ER to LL sows, 96 significantly differentially expressed known miRNAs (78 up-regulated and 18 down-regulated) were identified. The target gene expression and pathway enrichment analyses indicated that these DEMs may influence embryonic implantation by regulating pathways related to proliferation, immunization, and angiogenesis. Our findings help gain a better understanding of the role of miRNAs in the regulation of embryonic implantation and embryonic survival in pigs. Future studies to identify target mRNAs regulated by abundant miRNAs in the endometrium using a single type of endometrial cell (i.e., luminal or glandular epitheliums) will be critical to uncover their exact biological functions. 


\section{DATA AVAILABILITY}

The datasets used and analysed during the current study are available from the corresponding author on reasonable request. The raw reads produced in this study were deposited in the NCBI Sequence Read Archive (SRA), available using accession number PRJNA50573.

\section{ETHICS STATEMENT}

All researches involving animals were conducted according to animal ethics guidelines and approved by the Animal Care and Use Committee of South China Agricultural University (Guangzhou, China).

\section{AUTHOR CONTRIBUTIONS}

JL, ZW, and HZ designed the study. LH, RL, XQ, and SW performed the experiments, analyzed the data, and drafted the manuscript. XW performed the sequencing analysis. All authors read and approved the final manuscript.

\section{FUNDING}

This work was supported by the National Natural Science Foundation of China (31802033), the Guangdong Provincial Promotion Project on Preservation and Utilization of Local Breed of Livestock and Poultry (4300-F18260), and the Science \& Technology Planning Project of Guangzhou in China (201904010434). The funders had no role in study design, data

\section{REFERENCES}

Altmäe, S., Martinez-Conejero, J. A., Esteban, F. J., Ruiz-Alonso, M., StavreusEvers, A., Horcajadas, J. A., et al. (2013). microRNAs miR-30b, miR-30d, and miR-494 regulate human endometrial receptivity. Reprod. Sci. 20 (3), 308-317. doi: $10.1177 / 1933719112453507$

Aravin, A., and Tuschl, T. (2005). Identification and characterization of small RNAs involved in RNA silencing. FEBS Lett. 579 (26), 5830-5840. doi: 10.1016/j.febslet.2005.08.009

Ariel, R., Hanna, A., Juliet, S., Yoav, S., and Reuven, R. (2011). MicroRNAs are associated with human embryo implantation defects. Human Reprod. 26 (10), 2830-2840. doi: 10.1093/humrep/der255

Asangani, I. A., Rasheed, S. A., Nikolova, D., Leupold, J., Colburn, N., Post, S., et al. (2008). MicroRNA-21 (miR-21) post-transcriptionally downregulates tumor suppressor Pdcd4 and stimulates invasion, intravasation and metastasis in colorectal cancer. Oncogene 27 (15), 2128. doi: 10.1038/sj.onc.1210856

Ashworth, C., Pickard, A., Miller, S., Flint, A., and Diehl, J. (1997). Comparative studies of conceptus-endometrial interactions in Large White $\times$ Landrace and Meishan gilts. Reprod. Fertil. Dev. 9 (2), 217-226. doi: 10.1071/R96040

Audic, S., and Claverie, J.-M. (1997). The significance of digital gene expression profiles. Genome Res. 7 (10), 986-995. doi: 10.1101/gr.7.10.986

Bartel, D. P. (2004). MicroRNAs: genomics, biogenesis, mechanism, and function. Cell 116 (2), 281-297. doi: 10.1016/S0092-8674(04)00045-5

Bazer, F., Thatcher, W., Martinat-Botte, F., and Terqui, M. (1988). Conceptus development in Large White and prolific Chinese Meishan pigs. J. Reprod. Fertil. 84 (1), 37-42. doi: 10.1530/jrf.0.0840037

Bazer, F. W., and Johnson, G. A. (2014). Pig blastocyst-uterine interactions. Differentiation 87 (1), 52-65. doi: 10.1016/j.diff.2013.11.005 collection and analysis, decision to publish or preparation of the manuscript.

\section{ACKNOWLEDGMENTS}

We thank all the study participants, research staff, and students who assisted in animal sampling and technical support.

\section{SUPPLEMENTARY MATERIAL}

The Supplementary Material for this article can be found online at: https://www.frontiersin.org/articles/10.3389/fgene.2019.00661/ full\#supplementary-material

SUPPLEMENTARY FIGURE 1 | ClueGo network of pathways. Each node represents a pathway. The enrichment significance of pathway is reflected by the size of the nodes. Node color, represents the class that they belong. Mixed coloring means that the specific node belongs to multiple classes.

SUPPLEMENTARY TABLE 1 | Primer sequences for qPCR.

SUPPLEMENTARY TABLE 2 |The detailed sequence information in ER and LL small RNA libraries.

SUPPLEMENTARY TABLE 3 | Match_hairpin in ER library.

SUPPLEMENTARY TABLE 4 | Match_hairpin in LL library.

SUPPLEMENTARY TABLE 5 | Sequence editing of bases in the seed region of the miRNAsin ER and LL.

SUPPLEMENTARY TABLE 6 | Global expression profile of endometrial miRNAs on day 12 of gestation in LL and ER pigs.

SUPPLEMENTARY TABLE 7 | The differentially expressed miRNAs in ER and LL small RNA libraries.

Benjamini, Y., and Hochberg, Y. (1995). Controlling the false discovery rate: a practical and powerful approach to multiple testing. J R Stat Soc Series B Stat Methodol 57 (1), 289-300. doi: 10.1111/j.2517-6161.1995.tb02031.x

Bermont, L., Lamielle, F., Fauconnet, S., Esumi, H., Weisz, A., and Adessi, G. L. (2000). Regulation of vascular endothelial growth factor expression by insulin-like growth factor-I in endometrial adenocarcinoma cells. Int. J. Cancer 85 (1), 117-123. doi: 10.1002/(SICI)1097-0215(20000101)85:1<117::AID-IJC21>3.0.CO;2-X

Boyle, E. I., Weng, S., Gollub, J., Jin, H., Botstein, D., Cherry, J. M., et al. (2004). GO:: TermFinder-open source software for accessing Gene Ontology information and finding significantly enriched Gene Ontology terms associated with a list of genes. Bioinformatics 20 (18), 3710-3715. doi: 10.1093/bioinformatics/bth456

Carrington, J. C., and Ambros, V. (2003). Role of microRNAs in plant and animal development. Science 301 (5631), 336-338. doi: 10.1126/science.1085242

Chang, H. J., Lee, J. H., Hwang, K. J., Kim, M. R., Chang, K. H., Park, D. W., et al. (2008). Transforming growth factor (TGF)- $\beta 1$-induced human endometrial stromal cell decidualization through extracellular signal-regulated kinase and Smad activation in vitro: peroxisome proliferator-activated receptor gamma acts as a negative regulator of TGF- $\beta 1$. Fertil Steril 90 (4), 1357-1365. doi: 10.1016/j.fertnstert.2007.09.010

Chen, L., Yao, H., Wang, K., and Liu, X. (2017). Long non-coding RNA MALAT1 regulates ZEB1 expression by sponging miR-143-3p and promotes hepatocellular carcinoma progression. J. Cell. Biochem. 118 (12), 4836-4843. doi: $10.1002 /$ jcb. 26158

Cheng, J.-C., Chang, H.-M., and Leung, P. C. K. (2017). TGF- $\beta 1$ inhibits human trophoblast cell invasion by upregulating connective tissue growth factor expression. Endocrinology 158 (10), 3620-3628. doi: 10.1210/en.2017-00536

Christenson, R., Vallet, J., Leymaster, K., and Young, L. (1993). Uterine function in Meishan pigs. J. Reprod. Fertil. Suppl. 48, 279-289. 
Das, S., Chakraborty, I., Wang, J., Dey, S., and Hoffman, L. (1997). Expression of vascular endothelial growth factor (VEGF) and VEGF-receptor messenger ribonucleic acids in the peri-implantation rabbit uterus. Biol. Reprod. 56 (6), 1390-1399. doi: 10.1095/biolreprod56.6.1390

Feng, Y., Cao, J. H., Li, X. Y., and Zhao, S. H. (2011). Inhibition of miR-214 expression represses proliferation and differentiation of $\mathrm{C} 2 \mathrm{C} 12$ myoblasts. Cell Biochem. Funct. 29 (5), 378-383. doi: 10.1002/cbf.1760

Frankel, L. B., Christoffersen, N. R., Jacobsen, A., Lindow, M., Krogh, A., and Lund, A. H. (2008). Programmed cell death 4 (PDCD4) is an important functional target of the microRNA miR-21 in breast cancer cells. J. Biol. Chem. 283 (2), 1026-1033. doi: 10.1074/jbc.M707224200

Friedländer, M. R., Mackowiak, S. D., Li, N., Chen, W., and Rajewsky, N. (2011). miRDeep2 accurately identifies known and hundreds of novel microRNA genes in seven animal clades. Nucleic Acids Res. 40 (1), 37-52. doi: 10.1093/ nar/gkr688

Friedman, R., Farh, K., Burge C., and Bartel, D. (2008). Most mammalian mRNAs are conserved targets of microRNAs. Genome Res. 19 (1), 92-105. doi: 10.1101/ gr.082701.108

Geisert, R., Renegar, R., Thatcher, W., Roberts, R., and Bazer, F. (1982). Establishment of pregnancy in the pig: i. Interrelationships between preimplantation development of the pig blastocyst and uterine endometrial secretions. Biol. Reprod. 27 (4), 925-939. doi: 10.1095/biolreprod27.4.925

George, A. F., Rahman, K. M., Miller, D. J., Wiley, A. A., Camp, M. E., Bartol, F., et al. (2018). Effects of colostrum, feeding method, and oral IGF1 on porcine uterine development. Reproduction 155 (3), 259-271. doi: 10.1530/ REP-17-0658

Giles, F. J. (2001). The vascular endothelial growth factor (VEGF) signaling pathway: a therapeutic target in patients with hematologic malignancies. Oncologist 6 (Supplement 5), 32-39. doi: 10.1634/ theoncologist.6-suppl_5-32

Griffiths-Jones, S., Saini, H. K., van Dongen, S., and Enright, A. J. (2007). miRBase: tools for microRNA genomics. Nucleic Acids Res. 36 (suppl_1), D154-D158. doi: $10.1093 / \mathrm{nar} / \mathrm{gkm} 952$

Halder, J., Zhao, X., Soker, S., Paria, B., Klagsbrun, M., Das, S., et al. (2000). Differential expression of VEGF isoforms and VEGF164-specific receptor neuropilin-1 in the mouse uterus suggests a role for VEGF164 in vascular permeabilityandangiogenesisduringimplantation.Genesis26(3),213-224.doi: 10.1002/(SICI) 1526-968X(200003)26:3<213::AID-GENE7>3.0.CO;2-M

Haley, C., Lee, G., and Ritchie, M. (1995). Comparative reproductive performance in Meishan and Large White pigs and their crosses. Anim. Genet. 60 (2), 259267. doi: $10.1017 /$ S1357729800008420

Hannan, N., Paiva, P., Meehan, K., Rombauts, L., Gardner, D., and Salamonsen, L. (2011). Analysis of fertility-related soluble mediators in human uterine fluid identifies VEGF as a key regulator of embryo implantation. Endocrinology 152 (12), 4948-4956. doi: 10.1210/en.2011-1248

He, Z., Yi, J., Liu, X., Chen, J., Han, S., Jin, L., et al. (2016). MiR-143-3p functions as a tumor suppressor by regulating cell proliferation, invasion and epithelialmesenchymal transition by targeting QKI-5 in esophageal squamous cell carcinoma. Mol. Cancer 15 (1), 51. doi: 10.1186/s12943-016-0533-3

Hu, S.-J., Ren, G., Liu, J.-L., Zhao, Z.-A., Yu, Y.-S., Su, R.-W., et al. (2008). MicroRNA expression and regulation in mouse uterus during embryo implantation. J. Biol. Chem. 283 (34), 23473-23484. doi: 10.1074/jbc.M800406200

Inyawilert, W., Fu, T.-Y., Lin, C.-T., and Tang, P.-C. (2015). Let-7-mediated suppression of mucin 1 expression in the mouse uterus during embryo implantation. J. Reprod. Dev. 61 (2), 138-144. doi: 10.1262/jrd.2014-106

Jazdzewski, K., Liyanarachchi, S., Swierniak, M., Pachucki, J., Ringel, M. D., Jarzab, B., et al. (2009). Polymorphic mature microRNAs from passenger strand of premiR-146a contribute to thyroid cancer. Proc. Natl. Acad. Sci. U.S.A. 106 (5), 1502-1505. doi: 10.1073/pnas.0812591106

Kawahara, Y., Zinshteyn, B., Sethupathy, P., Iizasa, H., Hatzigeorgiou, A. G., and Nishikura, K. (2007). Redirection of silencing targets by adenosine-to-inosine editing of miRNAs. Science 315 (5815), 1137-1140. doi: 10.1126/science. 1138050

Langmead, B., Trapnell, C., Pop, M., and Salzberg, S. L. (2009). Ultrafast and memory-efficient alignment of short DNA sequences to the human genome. Genome Biol. 10 (3), R25. doi: 10.1186/gb-2009-10-3-r25

Laurent, L. (2010). MicroRNAs in embryonic stem cells and early embryonic development. J. Cell. Mol. Med. 12 (6A), 2181-2188. doi: 10.1111/j.1582-4934.2008.00513.x
Li, G., Li, Y., Li, X., Ning, X., Li, M., and Yang, G. (2011). MicroRNA identity and abundance in developing swine adipose tissue as determined by Solexa sequencing. J. Cell. Biochem. 112 (5), 1318-1328. doi: 10.1002/jcb.23045

Li, Q., Cheon, Y.-P., Kannan, A., Shanker, S., Bagchi, I. C., and Bagchi, M. K. (2004). A novel pathway involving progesterone receptor, 12/15-lipoxygenasederived eicosanoids, and peroxisome proliferator-activated receptor $\gamma$ regulates implantation in mice. J. Biol. Chem. 279 (12), 11570-11581. doi: 10.1074/jbc. M311773200

Li, M., Xia, Y., Gu, Y., Zhang, K., Lang, Q., Chen, L., et al. (2010). MicroRNAome of porcine pre-and postnatal development. PLoS One 5 (7), e11541. doi: 10.1371/ journal.pone.0011541

Li, W., Xi, Y., Xue, S., Wang, Y., Wu, L., Liu, H., et al. (2018). Sequence analysis of microRNAs during pre-implantation between Meishan and Yorkshire pigs. Gene 646, 20-27. doi: 10.1016/j.gene.2017.12.046

Lim, H., and Dey, S. K. (2000). PPAR $\delta$ functions as a prostacyclin receptor in blastocyst implantation. Trends Endocrinol. Metab. 11 (4), 137-142. doi: 10.1016/S1043-2760(00)00243-5

Lim, W., Bae, H., Bazer, F. W., and Song, G. (2018). Characterization of CC motif chemokine ligand 4 in the porcine endometrium during the presence of the maternal-fetal interface. Dev. Biol. 441 (1), 146-158. doi: 10.1016/j. ydbio.2018.06.022

Liu, R., Wang, M., Su, L., Li, X., Zhao, S., and Yu, M. (2015). The expression pattern of microRNAs and the associated pathways involved in the development of porcine placental folds that contribute to the expansion of the exchange surface area. Biol. Reprod. 93 (3), 1-13. doi: 10.1095/biolreprod.114.126540

Liu, W., Niu, Z., Li, Q., Pang, R. T., Chiu, P. C., and Yeung, W. S. (2016). MicroRNA and Embryo Implantation. Am. J. Reprod. Immunol. 75 (3), 263-271. doi: 10.1111/aji.12470

Liu, N., Okamura, K., Tyler, D. M., Phillips, M. D., Chung, W.-J., and Lai, E. C. (2008). The evolution and functional diversification of animal microRNA genes. Cell Res. 18 (10), 985. doi: 10.1038/cr.2008.278

Liu, W.-M., Pang, R. T., Cheong, A. W., Ng, E. H., Lao, K., Lee, K.-F., et al. (2012). Involvement of microRNA lethal-7a in the regulation of embryo implantation in mice. PLoS One 7 (5), e37039. doi: 10.1371/journal.pone.0037039

Liu, N., Tang, L.-L., Sun, Y., Cui, R.-X., Wang, H.-Y., Huang, B.-J., et al. (2013a). MiR-29c suppresses invasion and metastasis by targeting TIAM1 in nasopharyngeal carcinoma. Cancer Lett. 329 (2), 181-188. doi: 10.1016/j. canlet.2012.10.032

Liu, P., Qi, M., Ma, C., Lao, G., Liu, Y., Liu, Y., et al. (2013b). Let7a inhibits the growth of endometrial carcinoma cells by targeting Aurora-B. FEBS Lett. 587 (16), 2523-2529. doi: 10.1016/j.febslet.2013.05.065

Long, M., Wan, X., La, X., Gong, X., and Cai, X. (2015). miR-29c is downregulated in the ectopic endometrium and exerts its effects on endometrial cell proliferation, apoptosis and invasion by targeting c-Jun. Int. J. Mol. Med. 35 (4), 1119-1125. doi: 10.3892/ijmm.2015.2082

Lu, J., and Clark, A. G. (2012). Impact of microRNA regulation on variation in human gene expression. Genome Res. 22 (7), 1243-1254. doi: 10.1101/ gr.132514.111

Lv, C., Yu, W.-X., Wang, Y., Yi, D.-J., Zeng, M.-H., and Xiao, H.-M. (2018). MicroRNA-21 in extracellular vesicles contributes to the growth of fertilized eggs and embryo development in mice. Biosci. Rep. 38 (4), BSR20180036. doi: 10.1042/BSR20180036

Möller, B., Rasmussen, C., Lindblom, B., and Olovsson, M. (2001). Expression of the angiogenic growth factors VEGF, FGF-2, EGF and their receptors in normal human endometrium during the menstrual cycle. Mol. Hum. Reprod. 7 (1), 65-72. doi: 10.1093/molehr/7.1.65

Montazeri, M., Sanchez-Lopez, J., Caballero, I., Maslehat Lay, N., Elliott, S., and Fazeli, A. (2016). Interleukin-1 receptor antagonist mediates toll-like receptor 3 -induced inhibition of trophoblast adhesion to endometrial cells in vitro. Human Reprod. 31 (9), 2098-2107. doi: 10.1093/humrep/dew171

Montenegro, D., Romero, R., Kim, S.-S., Tarca, A. L., Draghici, S., Kusanovic, J. P., et al. (2009). Expression patterns of microRNAs in the chorioamniotic membranes: a role for microRNAs in human pregnancy and parturition. J. Pathol. Bacteriol. 217 (1), 113-121. doi: 10.1002/path.2463

Mu, S., Kang, B., Zeng, W., Sun, Y., and Yang, F. (2016). MicroRNA-143-3p inhibits hyperplastic scar formation by targeting connective tissue growth factor CTGF/ CCN2 via the Akt/mTOR pathway. Mol. Cell. Biochem. 416 (1-2), 99-108. doi: 10.1007/s11010-016-2699-9 
Nicoli, S., Standley, C., Walker, P., Hurlstone, A., Fogarty, K. E., and Lawson, N. D. (2012). MicroRNA-mediated integration of haemodynamics and VEGF signalling during angiogenesis. Nature 464 (7292), 1196. doi: 10.1038/nature08889

Nielsen, M., Hansen, J., Hedegaard, J., Nielsen, R., Panitz, F., Bendixen, C., et al. (2010). MicroRNA identity and abundance in porcine skeletal muscles determined by deep sequencing. Anim. Genet. 41 (2), 159-168. doi: 10.1111/j.1365-2052.2009.01981.x

Park, S., Lim, W., Bazer, F. W., and Song, G. (2017). Naringenin induces mitochondriamediated apoptosis and endoplasmic reticulum stress by regulating MAPK and AKT signal transduction pathways in endometriosis cells. MHR: Basic Sci. Reprod. Med. 23 (12), 842-854. doi: 10.1093/molehr/gax057

Pope, W. F., and First, N. L. (1985). Factors affecting the survival of pig embryos. Theriogenology 23 (1), 91-105. doi: 10.1016/0093-691X(85)90075-5

Qian, K., Hu, L., Chen, H., Li, H., Liu, N., Li, Y., et al. (2009). Hsa-miR-222 is involved in differentiation of endometrial stromal cells in vitro. Endocrinology 150 (10), 4734-4743. doi: 10.1210/en.2008-1629

Rosenbluth, E. M., Shelton, D. N., Sparks, A. E. T., Eric, D., Lane, C., and Van Voorhis, B. J. (2013). MicroRNA expression in the human blastocyst. Fertil. Steril. 99 (3), 855-861.e853. doi: 10.1016/j.fertnstert.2012.11.001

Scofield, A. M., Clegg, F. G., and Lamming, G. E. (1974). Embryonic mortality and uterine infection in the pig. J. Reprod. Fertil. 36 (2), 353-361. doi: 10.1530/ jrf. 0.0360353

Shigematsu, S., Yamauchi, K., Nakajima, K., Iijima, S., Aizawa, T., and Hashizume, K. (1999). IGF-1 regulates migration and angiogenesis of human endothelial cells. Endocr. J. 46 (Suppl), S59-S62. doi: 10.1507/endocrj.46.Suppl_S59

Song, T., Zhao, J., Jiang, T., Jin, X., Li, Y., and Liu, X. (2018). Formononetin protects against balloon injury-induced neointima formation in rats by regulating proliferation and migration of vascular smooth muscle cells via the TGF- $\beta 1 /$ Smad3 signaling pathway. Int. J. Mol. Med. 42 (4), 2155-2162. doi: 10.3892/ ijmm.2018.3784

Spencer, T. E., Johnson, G. A., Bazer, F. W., Burghardt, R. C., and Palmarini, M. (2006). Pregnancy recognition and conceptus implantation in domestic ruminants: roles of progesterone, interferons and endogenous retroviruses. Reprod. Fertil. Dev. 19 (1), 65-78. doi: 10.1071/RD06102

Stroband, H., Voolstra, C., Taverne, N., and Hazeleger, W. (1992). Morphological indications of secretion by uterine epithelial cells and changes in the ratio between acidic and basic uterine proteins during early pregnancy in Chinese Meishan pig. Eur J Morphol 30 (3), 205-218.

Su, L., Zhao, S., Zhu, M., and Yu, M. (2010). Differential expression of microRNAs in porcine placentas on days 30 and 90 of gestation. Reprod. Fertil. Dev. 22 (8), 1175-1182. doi: 10.1071/RD10046

Tang, R., Yang, C., Ma, X., Wang, Y., Luo, D., Huang, C., et al. (2016). MiR-let-7a inhibits cell proliferation, migration, and invasion by down-regulating PKM2 in gastric cancer. Oncotarget 7 (5), 5972. doi: 10.18632/oncotarget.6821

Tsang, H., Cheung, T.-Y., Kodithuwakku, S. P., Chai, J., Yeung, W. S., Wong, C. K., et al. (2013). Perfluorooctanoate suppresses spheroid attachment on endometrial epithelial cells through peroxisome proliferator-activated receptor alpha and down-regulation of Wnt signaling. Reprod. Toxicol. 42, 164-171. doi: 10.1016/j. reprotox.2013.08.001

Wagner, G. P., Kin, K., and Lynch, V. J. (2012). Measurement of mRNA abundance using RNA-seq data: RPKM measure is inconsistent among samples. Theory Biosci. 131 (4), 281-285. doi: 10.1007/s12064-012-0162-3
Wang, C.-M., Wang, Y., Fan, C.-G., Xu, F.-F., Sun, W.-S., Liu, Y.-G., et al. (2011). miR-29c targets TNFAIP3, inhibits cell proliferation and induces apoptosis in hepatitis B virus-related hepatocellular carcinoma. Biochem. Biophys. Res. Commun. 411 (3), 586-592. doi: 10.1016/j.bbrc.2011.06.191

Wen, M., Shen, Y., Shi, S., and Tang, T. (2012). miREvo: an integrative microRNA evolutionary analysis platform for next-generation sequencing experiments. BMC Bioinformatics 13 (1), 140. doi: 10.1186/1471-2105-13-140

Wessels, J. M., Edwards, A. K., Khalaj, K., Kridli, R. T., Bidarimath, M., and Tayade, C. (2013). The microRNAome of pregnancy: deciphering miRNA networks at the maternal-fetal interface. PLoS One 8 (11), e72264. doi: 10.1371/ journal.pone.0072264

Wilson, M. E., Biensen, N. J., and Ford, S. P. (1999). Novel insight into the control of litter size in pigs, using placental efficiency as a selection tool. J. Anim. Sci. 77 (7), 1654-1658. doi: 10.2527/1999.7771654x

Wu, L., Fan, J., and Belasco, J. G. (2006). MicroRNAs direct rapid deadenylation of mRNA. Proc. Natl. Acad. Sci. U.S.A. 103 (11), 4034-4039. doi: 10.1073/ pnas.0510928103

Yang, T.-S., Yang, X.-H., Wang, X.-D., Wang, Y.-L., Zhou, B., and Song, Z.-S. (2013). MiR-214 regulate gastric cancer cell proliferation, migration and invasion by targeting PTEN. Cancer Cell Int. 13 (1), 68. doi: 10.1186/1475-2867-13-68

Youngs, C., Christenson, L., and Ford, S. (1994). Investigations into the control of litter size in swine: III. A reciprocal embryo transfer study of early conceptus development. J. Anim. Sci. 72 (3), 725-731. doi: 10.2527/1994.723725x

Youngs, C., Ford, S., McGinnis, L., and Anderson, L. (1993). Investigations into the control of litter size in swine: I. Comparative studies on in vitro development of Meishan and Yorkshire preimplantation embryos. J. Anim. Sci. 71 (6), 15611565. doi: 10.2527/1993.7161561x

Zavy, M. T., and Geisert, R. D. (1994). Embryonic mortality in domestic species. FL: CRC Press Boca Raton.

Zhang, Z. (1986). Chinese pig breed records. Shanghai, China: Shanghai Science and Technology Press.

Zhang, G., Cheng, Y., Zhang, Q., Li, X., Zhou, J., Wang, J., et al. (2018). ATX-LPA axis facilitates estrogen-induced endometrial cancer cell proliferation via MAPK/ERK signaling pathway. Mol. Med. Rep. 17 (3), 4245-4252. doi: 10.3892/mmr.2018.8392

Zhang, H., Wang, S., Liu, M., Zhang, A., Wu, Z., Zhang, Z., et al. (2013). Differential gene expression in the endometrium on gestation day 12 provides insight into sow prolificacy. BMC Genomics 14 (1), 45. doi: 10.1186/1471-2164-14-45

Zhang, Z.-C., Li, Y.-Y., Wang, H.-Y., Fu, S., Wang, X.-P., Zeng, M.-S., et al. (2014). Knockdown of miR-214 promotes apoptosis and inhibits cell proliferation in nasopharyngeal carcinoma. PLoS One 9 (1), e86149. doi: 10.1371/journal. pone.0086149

Conflict of Interest Statement: The authors declare that the research was conducted in the absence of any commercial or financial relationships that could be construed as a potential conflict of interest.

Copyright (C) 2019 Hong, Liu, Qiao, Wang, Wang, Li, Wu and Zhang. This is an openaccess article distributed under the terms of the Creative Commons Attribution License (CC BY). The use, distribution or reproduction in other forums is permitted, provided the original author(s) and the copyright owner(s) are credited and that the original publication in this journal is cited, in accordance with accepted academic practice. No use, distribution or reproduction is permitted which does not comply with these terms. 